\title{
The $\sigma$ Problem of the Crab Pulsar Wind
}

\author{
J. G. Kirk \\ Max-Planck-Institut für Kernphysik, Postfach 1039 80, \\ 69029 Heidelberg, Germany \\ O. Skjæraasen
}

Observatoire de Strasbourg, 11 rue de l'Université, F-67000 Strasbourg, France

\begin{abstract}
The conversion of the Crab pulsar wind from one dominated by Poynting flux close to the star to one dominated by particle-borne energy at the termination shock is considered. The idea put forth by Coroniti (1990) and criticized by Lyubarsky \& Kirk (2001) that reconnection in a striped wind is responsible, is generalized to include faster prescriptions for the a priori unknown dissipation rate. Strong acceleration of the wind is confirmed, and the higher dissipation rates imply complete conversion of Poynting flux into particle-borne flux within the unshocked wind.
\end{abstract}

\section{Introduction}

A puzzling feature of the relativistic MHD wind of the Crab pulsar is that it appears to arrive at its termination shock as a particle dominated flow (Rees \& Gunn 1974; Kennel \& Coroniti 1984; Emmering \& Chevalier 1987). This is difficult to understand, because the wind almost certainly carries its energy by Poynting flux close to the star, and, given that relativistic MHD winds collimate only weakly (Beskin, Kuznetsova \& Rafikov 1998; Chiueh, Li \& Begelman 1998; Bogovalov \& Tsinganos 1999; Lyubarsky \& Eichler 2001; Lyubarsky 2002) it is not obvious how the transformation to particle-borne flux can occur, or why it is apparently not observed. The acceleration intrinsic to axisymmetric forcefree winds (Buckley 1977; Contopoulos \& Kazanas 2002) does not alleviate the difficulty, since the flow is still magnetically dominated outside the sonic point where such solutions lose their validity.

An obliquely rotating neutron star drives a wind which, near the equatorial plane, consists of alternating stripes of magnetic field of opposite polarity. The magnetic field is linked alternately to the open field lines emerging from different magnetic hemispheres, as these are swept around the rotational equator. This led Coroniti (1990) to suggest that reconnection or, more accurately, dissipation at the current sheet separating the alternating polarities, might effect the required conversion. He estimated the dissipation rate by assuming the thickness of the current sheet was approximately equal to the gyro radius of the hot particles it contained and found that the magnetic field could be annihilated 
before the wind reached the termination shock. However, Coroniti's treatment neglected the acceleration of the wind flow. Using the same micro-physics estimate for dissipation, Lyubarsky \& Kirk (2001) showed that this effect results in an annihilation rate far too small to be effective.

Kirk \& Skjæraasen (2003) recently identified the dissipation estimate of Coroniti as a lower limit and examined the effect of alternative micro-physics prescriptions, including an upper limit on the dissipation rate. Their findings, which we paraphrase in this paper, re-open the possibility that the conversion of Poynting flux to particle-borne flux occurs through dissipation of magnetic field energy at the current sheet of a striped wind.

\section{Small Wavelength Approximation and Dissipation Prescriptions}

The approach of Lyubarsky \& Kirk (2001) and Kirk \& Skjæraasen (2003) is based on computing the slow evolution of an entropy wave as it is convected outwards by the stellar wind. The method employs a separation of length scales into a short one, identified with the wavelength of the magnetic field reversals, and a long one, identified with the radius, i.e., the scale on which the density changes as a result of the spherically expanding flow. The equations of conservation of particles and energy/momentum are then ordered in the small parameter formed from the ratio of these lengths. The entropy wave whose evolution is sought can be described in several ways. One is in terms of the parameters which describe the Harris sheath (Harris 1962; Hoh 1968); another is in terms of the parameters of two uniform fluid layers - one hot and unmagnetized, the other cold and magnetized - separated from each other by discontinuities in density, velocity and magnetic field, but having equal pressures. Each of these configurations satisfies the zeroth order conservation equations, indicating that the wave does not evolve on this short length-scale.

On the long length scale, however, evolution of the wave is possible. It is computed by regularizing the expansion, i.e., by demanding that the secular terms in the first-order equations vanish. The conservation equations by themselves are not sufficient to solve for all the parameters of the wave as a function of radius - an additional constraint is required, reflecting the need for new physics input to determine the rate at which dissipation proceeds. In MHD simulations, the new input needed to describe dissipation is a functional relation between the current and the electric field: an anomalous conductivity. For our computations, however, it suffices to prescribe one of the wave parameters (the thickness of the Harris sheath, for example) in terms of the others.

The Coroniti (1990) prescription implies a relative drift speed of the oppositely charged species roughly equal to that of light. In other words, there exists no Harris equilibrium for a sheath thinner than that assumed by Coroniti. The thinner the sheet, the slower the dissipation, so that Coroniti's prescription provides a lower limit on the dissipation rate. Kirk \& Skjæraasen (2003) consider two alternative prescriptions: that the sheath thickness is governed by the (linear) growth rate of the tearing instability, as suggested by Lyubarsky (1996), and that the expansion speed of the sheath is limited to its internal sound speed. The motivation for the latter choice stems from the observation that only the oscillating component of the magnetic field in the entropy wave can be dissipated. 

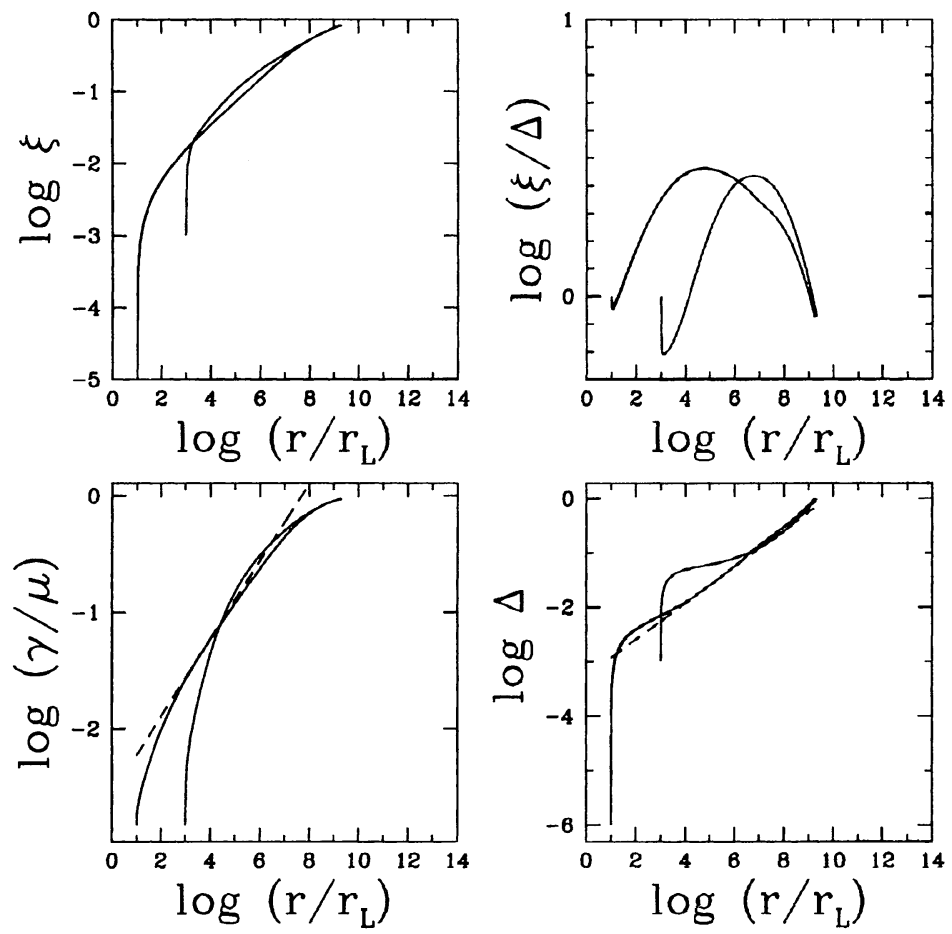

Figure 1. The Crab pulsar wind with maximum rate of dissipation (sonic sheath expansion) and particle injection rate into the Nebula of $3 \times 10^{40} \mathrm{~s}^{-1}$. Shown as functions of $r / r_{\mathrm{L}}$, (where $r_{\mathrm{L}}$ is the radius of the light cylinder) are the ratio $\xi$ of the particle density in the current sheet to that in the magnetized wind, the Lorentz factor $\gamma$ in units of its maximum value $\mu\left(=2 \times 10^{4}\right.$ in this case $)$, the thickness $\Delta$ of the sheath in units of $\pi r_{\mathrm{L}}$ and the ratio $\xi / \Delta$. The termination shock is located at $\log \left(r / r_{\mathrm{L}}\right) \approx 9$.

This suggests that the boundaries of the hot fluid must be in causal contact in order to guarantee that they advance into regions of opposite magnetic polarity at equal speeds. Thus, expansion at the internal sound speed provides an upper limit to the dissipation rate.

\section{Implications for the Crab Pulsar Wind}

The dissipation of the magnetic field in the wind of the Crab pulsar depends on the particle load carried by the wind. For a particle loss rate of $\dot{N}<6 \times 10^{38} \mathrm{~s}^{-1}$, the theoretical upper limit on the dissipation rate lies below the lower limit given by the Coroniti prescription, so that the treatment is inconsistent. In this case, slow evolution of the entropy wave is impossible. Presumably the system adjusts by either becoming unsteady or by steadily converting the flux into a 
mode which propagates in the fluid frame (e.g., an electromagnetic mode). For $6 \times 10^{38} \mathrm{~s}^{-1}<\dot{N}<3 \times 10^{40} \mathrm{~s}^{-1}$ none of the three dissipation prescriptions succeeds in converting significant amounts of Poynting flux before the termination shock is encountered. For $\dot{N}>3 \times 10^{40} \mathrm{~s}^{-1}$, however, dissipation at the fastest permitted rate, as depicted in Figure 1, results in complete conversion before the termination shock is encountered. This rather high level of pair injection into the Nebula cannot be explained in terms of current theories of cascade formation in the inner magnetosphere (Hibschmann \& Arons 2001), but is nevertheless consistent with the observed number of radio-emitting electrons (Gallant et al. 2002).

Can the conversion of Poynting flux to particle-borne flux be observed directly? Arons (1979) noticed that conditions in the wind will make such a signal pulse at the rotation frequency of the star. The obvious implication that the optical to gamma-ray pulses are indeed the signature of this conversion is further supported by the approximate correspondence of the sheet geometry to the observed pulse form (Kirk, Skjæraasen \& Gallant 2002).

Acknowledgments. We thank Yves Gallant and Yuri Lyubarsky for helpful discussions.

\section{Reîerences}

Arons, J. 1979, Space Sci. Rev., 24, 437

Beskin, V. S., Kuznetsova, I. V., \& Rafikov, R. R. 1998, MNRAS, 299, 341

Bogovalov, S., \& Tsinganos, K. 1999, MNRAS, 305, 211

Buckley, R. 1977, MNRAS, 180, 125

Chiueh, T., Li, Z.-Y., \& Begelman, M. C. 1998, ApJ, 505, 835

Contopoulos, I., \& Kazanas, D. 2002, ApJ, 566, 336

Coroniti, F. V. 1990, ApJ, 349, 538

Emmering, R. T., \& Chevalier, R. A. 1987, ApJ, 321, 334

Gallant, Y. A., van der Swaluw, E., Kirk, J. G., \& Achterberg, A. 2002, in ASP Conf. Ser., Vol. 271, Neutron Stars in Supernova Remnants, eds. P. O. Slane, \& B. M. Gaensler, (San Francisco: ASP), p. 99

Harris, E. G. 1962, Nuovo Cimento, 23, 115

Hibschman, J. A., \& Arons, J. 2001, ApJ 560, 871

Hoh, F. C. 1968, Physics Fluids, 9, 277

Kennel, C. F., \& Coroniti, F. V. 1984, ApJ, 283, 694

Kirk, J. G., \& Skjæraasen, O. 2003, ApJ, 591, 366

Kirk, J. G., Skjæraasen, O., \& Gallant, Y. A. 2002, A\&A, 388, L29

Lyubarsky, Y. 1996, A\&A, 311, 172

- 2002, MNRAS, 329, L34

Lyubarsky, Y., \& Eichler, D. 2001, ApJ, 562, 494

Lyubarsky, Y., \& Kirk, J. G. 2001, ApJ, 547, 437

Rees, M. J., \& Gunn, J. E. 1974, MNRAS, 167, 1 